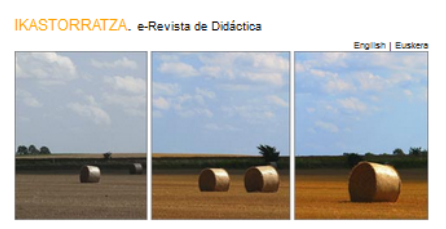

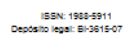

Inicio, Sobre nosotros, Publicacionesı, Participa

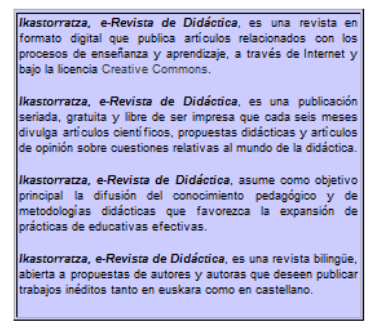

\section{IKASTORRATZA. Didaktikarako e-aldizkaria}

\author{
IKASTORRATZA. e-journal on Didactics
}

ISSN: 1988-5911 (Online) Journal homepage: http://www.ehu.eus/ikastorratza/

Percepción de estudiantes universitarios sobre su formación académica en matemática y estadística

\author{
IRASTORRATZA. e-Revista de \\ Didáctica
}

\author{
Ivett Reyes Guillén \\ reyes.flor@unach.mx \\ Socorro Fonseca Córdoba \\ socorro.cordoba@unach.mx \\ Miguel Ángel Gómez López \\ miguel.gomez@unach.mx
}

To cite this article:

Reyes, I., Fonseca, S. \& Gómez, M.A. (2021). Percepción de estudiantes universitarios sobre su formación académica en matemática y estadística. IKASTORRATZA. e-Revista de Didáctica, 26, 116-132. DOI: 10.37261/26_alea/6

To link to this article:

https://doi.org/10.37261/26_alea/6

Published online: 31 March 2021 


\title{
Percepción de estudiantes universitarios sobre su formación académica en matemática y estadística
}

\section{University students' perception of their academic training on mathematics and statistics}

\author{
Ivett Reyes Guillén ${ }^{1}$, Socorro Fonseca Córdoba ${ }^{2}$ y Miguel Ángel Gómez López
}

${ }^{1}$ Facultad de Ciencias Sociales, Universidad Autónoma de Chiapas, México, Cuerpo Académico Sociedad, Cultura y Educación reyes.flor@unach.mx

${ }^{2}$ Facultad de Ciencias Sociales, Universidad Autónoma de Chiapas, México, Cuerpo Académico Sociedad, Cultura y Educación socorro.cordoba@unach.mx

${ }^{3}$ Facultad de Ciencias Sociales, Universidad Autónoma de Chiapas, México miguel.gomez@unach.mx

\section{Resumen}

El presente artículo muestra los resultados de una investigación que tuvo como objetivo analizar las percepciones de estudiantes de la Licenciatura en Economía, sobre el aprendizaje de la matemática, los procesos de enseñanza y la comprensión del idioma español en que son impartidas las clases. La metodología utilizada fue de tipo descriptivo, no experimental, transversal y de campo. Como resultados, los estudiantes perciben a la matemática como tensa; pero atractiva, con procesos de enseñanza que, en ocasiones, no son muy claros. La enseñanza-aprendizaje exitosa en matemáticas y las disciplinas que derivan de ella son un desafío independientemente del nivel académico. Se considera necesario realizar estudios que reflejen la relación de esta situación con variables como la barrera idiomática.

Palabras clave: Percepciones, matemáticas, barrera idiomática, educación universitaria, investigación cualitativa. 


\begin{abstract}
This article shows the results of research to analyze the perceptions of students of the bachelor's degree in Economics, about learning mathematics, teaching processes, and understanding the Spanish language in which the classes are taught. The methodology used was descriptive, non-experimental, cross-cutting and field. As results, students perceive mathematics as tense; but attractive, with teaching processes that are sometimes not noticeably clear. Successful teaching-learning in mathematics and the disciplines that derive from it are a challenge regardless of academic level. Studies reflecting the relationship of this situation to variables such as language barriers are considered necessary.
\end{abstract}

Key words: Perceptions, mathematics, language barrier, university education. qualitative research. 


\section{Introducción}

En el presente estudio, abordaremos la exposición de resultados de un estudio realizado para conocer las percepciones que estudiantes universitarios tienen, con relación a su formación matemática y estadística.

Desde el punto de vista tradicional, la educación ha sido considerada como un conjunto de procesos socioculturales, y es a través de ellos que las distintas generaciones han sido informadas y educadas, asegurando la asimilación de conocimientos en los contextos o grupos poblacionales a los que pertenecen (Durkheim, 1979; Flegg et al., 2012). Es por ello por lo que, la pedagogía se presenta como una herramienta para facilitar a los procesos de enseñanza-aprendizaje y podamos reflexionar sobre esto en el campo intelectual de la educación, que, en el caso que nos ocupa, nos lleva a reflexionar sobre procesos de formación universitaria (Zuluaga et al., 2003; Cossio, 2014).

A la formación universitaria, se le considera como un asunto complejo con procesos culturales, sociales, políticos, económicos como factores incidentes, es decir, que influyen en el proceso y en la efectividad de este (Wismath y Worral, 2015; LárezVillarroel, 2018). Como menciona Gadamer (Gadamer, 1984; Catoggio, 2007) la formación es uno de los conceptos base del humanismo moderno, que va más allá de moldear a los sujetos, estando insertos el aprendizaje, la enseñanza y el desarrollo personal facilitando los procesos de autoconstrucción. Lo anterior, coincide con lo expuesto en distintos momentos por Bollnow (2001), Taborda y Buriticá (2007); García (2014) y Villa et al (2015) quienes agregan a la autoformación y a la experiencia, como elementos que vuelven complejos a los sujetos, naturaleza reflejada de principio a fin en los procesos educativos.

Ahora bien, la matemática, como disciplina, tiene importancia elemental dentro del desarrollo, la cultura, ciencia y tecnología de todos los tiempos, facilitando la generación del pensamiento lógico y racional (Dou, 1970; Courant y Robins, 1976; Grafe, 1990, Uzuriaga et al., 2012; Martínez, 2014; Sánchez y Camacho, 2017; Cerda et al., 2017). Por ello, a lo largo de la formación educativa de los sujetos, la formación matemática es un eje transversal y aportará elementos para el correcto desempeño profesional dentro de una sociedad en dinámica constante. 
Por otra parte, cuando hablamos de procesos globales, en la actualidad hablamos de la interacción mundial en procesos de la vida cotidiana, y estas interacciones ofrecen escenarios donde la diversidad de grupos étnicos se hace presente participando dentro de un mismo proceso.

Al hablar de la enseñanza de la matemática, implica procesos pedagógicos que requieren de la uniformidad del lenguaje, tanto técnico como de comprensión del idioma en que se realice la enseñanza y la de recepción de los educandos.

Al respecto, Rivera y Alzate (2012), así como Pamplona et al. (2019) consideran como factores que facilitan el quehacer docente y la participación del estudiante a la buena escucha, motivación permanente, promoción del trabajo en equipo y participación creadora, generándose un ambiente de trabajo educativo agradable, respetuoso y de buena comunicación, en ambientes con diversidad cultural.

Por lo anterior y en aulas donde interactúan diferentes culturas, es muy útil contar con herramientas didácticas que permitan a los estudiantes incluirse en procesos educativos, en este caso matemáticos, que faciliten su entendimiento e interpretación. Todo ello, partiendo del entendimiento de la matemática como una ciencia que puede atenderse desde un pensamiento culturalmente incluyente. Esto genera riqueza educativa, respeto a la igualdad de derechos y oportunidades en la educación.

Dentro de este escenario de diversidad étnica y cultural, localizamos a las dificultades en la interpretación lingüística, que influyen en el aprendizaje de conceptos básicos matemáticos, así como de las indicaciones para la resolución de problemas. Esta realidad nos lleva a reflexionar sobre la importancia de incluir el pensamiento matemático en nuestras aulas y generar espacios proactivos y asertivos en la enseñanza de esta disciplina. La matemática se expresará entonces desde lo universal de su existencia.

En contextos educativos con diversidad cultural, la docencia no es tarea sencilla, es necesario tomar en cuenta esta realidad, investigarla y generar información que facilite el aprendizaje para todos. La realidad nos muestra posturas que ignoran estas necesidades y consideran la importancia de atender a la diversidad cultural de los alumnos. No obstante, hay una tercera postura que hace referencia a la atención de procesos homogeneizadores (Martínez, 2002; López, 2010; Flegg et al., 2012; Rodríguez, 2017). Esta última parecería un tanto limitante al verdadero desarrollo y genera procesos de violencia dentro de la 
enseñanza-aprendizaje; pero es común en las actividades cotidianas de la educación universitaria con modos educativos tradicionalistas.

Atender a la realidad, exige ideas, conocimientos, currículo y sobre todo atención al sujeto de la educación. Si bien, se puntualizan estas necesidades, también es claro que requiere de conciencia, humanidad y creatividad tanto de los investigadores dentro del área de la educación, como de los docentes en el área de la praxis educativa. Estos tres elementos lograrán evitar lo que para Knijnik (2007) es la parodia de lo cotidiano. Es decir, evitar caer en la enseñanza de la matemática en papel, sino más bien, acompañar al estudiante para comprender la aplicación de esta disciplina en la vida cotidiana.

De esta manera, en el estudio se exploraron las percepciones tanto del nivel educativo que cursan en la actualidad, como de niveles educativos previos. El estudio fue realizado en Chiapas, México, estado diverso en cuanto a los grupos étnicos que la conforman; por lo que, el análisis realizado se aborda desde la perspectiva pedagógica y matemática.

\section{Métodos}

La metodología utilizada en este trabajo se planteó de tipo descriptivo, no experimental, transversal y de campo. Es de carácter cualitativo, fenomenológica, pues no pretende la comprobación de teorías. Su intención es analizar las percepciones de estudiantes de la Licenciatura en Economía, sobre el aprendizaje de la matemática, los procesos de enseñanza y la comprensión del idioma español en que son impartidas las clases.

El estudio se realizó en la Licenciatura en Economía de Facultad de Ciencias Sociales, Universidad Autónoma de Chiapas (UNACH), en México. Los datos se obtuvieron a través de un cuestionario aplicado a un grupo de 100 estudiantes. Es importante mencionar, por la naturaleza del estudio, que el 30\% de estudiantes tienen al tzeltal y tzotzil como lengua materna de origen mayense; no dominan el español y las clases de todas las asignaturas en el programa de Licenciatura en Economía, se imparten en el idioma español.

En el estudio, se exploraron las percepciones tanto del nivel educativo que cursan en la actualidad, como de niveles educativos previos. Para lograr lo anterior, el instrumento fue un cuestionario (escala Likert) de percepción acerca de los factores pedagógicos, 
psicológicos, antropológicos y sociológicos que pueden incidir en la comprensión del área. Se aplicó mediante un muestreo aleatorio a los alumnos de la Licenciatura en Economía de la Facultad mencionada.

El cuestionario se compone de 20 ítems y se estructura en los siguientes apartados: a) datos generales; b) percepción de la matemática como asignatura; c) percepción de la matemática en niveles previos a la universidad; d) percepción de la estadística/matemática, sus docentes y el idioma en que imparten las clases. Se determinó la confiabilidad; utilizando el coeficiente de Alpha de Cronbach de consistencia interna. Este proceso de validación consiste en la fundamentación de la confiabilidad del cuestionario y de procedimientos de validez estructural de los reactivos con relación a su área de clasificación. El coeficiente puede tomar valores de 0 y 1, considerando normalmente a los coeficientes superiores a 0.7 como los más confiables (Nunnally, 1967). En este estudio, después de la validación mediante prueba piloto, se obtuvo 0.65; se observaron las medianas, medida de tendencia central recomendada para escalas ordinales.

El diseño metodológico tuvo tres momentos:

1. Selección de la muestra. Se seleccionaron de forma aleatoria a 100 estudiantes de la Licenciatura en Economía de la Facultad de Ciencias Sociales, UNACH.

2. Diseño del instrumento. Para el diseño del cuestionario (tipo Lickert), se consideraron estudios relacionados con las percepciones y concepciones sobre la matemática y la estadística, entre ellos citamos a Hassad (2011), Estrada et al (2011) y Devlin (2008). Dentro de la estructura del cuestionario se tomaron en cuenta los siguientes apartados: a) datos generales; b) percepción de la matemática como asignatura; c) percepción de la matemática en niveles previos a la universidad; d) percepción de la estadística/matemática, sus docentes y el idioma en que imparten las clases. El cuestionario consta de 20 ítems con 4 niveles de respuesta.

3. Aplicación de la prueba piloto. Se aplicaron 20 cuestionarios como prueba piloto del mismo, a estudiantes universitarios de otras licenciaturas en la UNACH. Al término de esta, se analizaron los elementos de mejora en la redacción de los ítems y se fortaleció el cuestionario. 
4. Aplicación del cuestionario. El proceso se realizó bajo consentimiento informado de los participantes. Se obtuvo una muestra de 100 estudiantes, con participación de ambos sexos y con representación de los semestres activos durante el período enero-junio 2020. Dadas las condiciones de confinamiento por COVID-19, la aplicación fue virtual, a través de correo electrónico de cada estudiante.

\section{Resultados}

\subsection{Datos generales de los encuestados.}

Los jóvenes participaron contestando el cuestionario fueron 100 estudiantes de la Licenciatura en Economía de la Facultad de Ciencias Sociales de la UNACH. La edad promedio de los participantes de la encuesta: 19 años.

En cuanto al lugar de procedencia, 36\% son jóvenes de San Cristóbal de Las Casas, 14\% de Tuxtla Gutiérrez, 8\% de Tenejapa y 42\% de distintos municipios del estado de Chiapas. Al respecto, es importante mencionar que San Cristóbal y Tenejapa, están ubicados en la Zona Altos del estado, con alta presencia de población indígena tzeltal y tzotzil. Tuxtla Gutiérrez es la capital del estado, y se localiza a escasos 30 minutos de trasportación vía terrestre de San Cristóbal. Son, además, poblaciones con alta y muy alta marginación socioeconómica, áreas de concentración de población de comunidades altamente marginadas que migran buscando elevar su calidad de vida. El 100\% de ellos viven actualmente en San Cristóbal por ser el lugar donde se imparte la Licenciatura en Economía.

La lengua materna es una variable lingüística que sostiene en un porcentaje representativo, 20\%, a la lengua indígena (variantes mayenses: tzeltal, tzotzil, chol y zoque). El resto corresponde al español como lengua materna de los encuestados.

\subsection{Percepción de la estadística como asignatura después de haberla cursado.}

La estadística es percibida como una asignatura bastante agradable (70\%), no obstante, el $30 \%$ la percibe como algo agradable; aun existiendo la respuesta nada agradable, nadie la eligió. Paradójicamente, pero como parte de los resultados, preguntando como lo 
atractiva que les parece la asignatura, 56\% la consideran muy atractiva, mientras que 38\% solo algo atractiva y 6\% la considera repulsiva.

Así también, en cuanto al grado de complejidad, un grupo de jóvenes la consideran compleja (58\%) y otro grupo la percibe como simple o sencilla (42\%).

Ahora bien, respecto a la percepción de utilidad de la asignatura de estadística, 90\% la considera de gran utilidad y un $10 \%$ la considera inútil. Y en cuanto a la actividad con la que se desarrolla la asignatura dentro del programa de licenciatura en economía, $80 \%$ la perciben como muy activa y $20 \%$ solo algo activa.

Según el grado de tensión de la Estadística como asignatura, los resultados arrojan que se percibe como algo tensa en un 48\%, muy tensa en el 6\% y $46 \%$ la perciben como relajada.

En cuanto a la claridad de los contenidos temáticos de la Estadística, 90\% los perciben como claros y muy claros; mientras que el 10\% restante los perciben como confusos y bastante confusos.

\subsection{Percepción de universitarios respecto a su aprendizaje de la matemática en nivel primaria, secundaria y bachillerato.}

\subsubsection{Primaria}

Con relación a los contenidos temáticos en nivel primaria son algo confusos (50\%), bastante claros (28\%), algo claros (22\%).

En cuanto a su nivel de aprendizaje, algo, suficiente o mucho, el porcentaje mayoritario considera que aprendió algo (50\%), seguido de aprendió mucho (36\%) y el porcentaje restante percibe haber aprendido lo suficiente (14\%).

\subsubsection{Secundaria}

Consideran que los contenidos temáticos en nivel secundaria son bastante claros (44\%), algo claros (26\%) algo confusos (22\%), bastante confusos (8\%).

La percepción sobre el aprendizaje que obtuvieron sobre matemáticas en nivel secundaria, el 54\% considera haber aprendido algo, 28\% aprendido mucho, 10\% aprendido lo suficiente y $8 \%$ no haber aprendido de la matemática en ese nivel. 


\subsubsection{Bachillerato o preparatoria}

Los contenidos en el área de la matemática a nivel preparatoria son percibidos como bastante claros en un 60\%; algo claros en $24 \%$ y $16 \%$ bastante confusos. Lo anterior lleva a considerar que aprendieron algo (54\%), mucho (38\%), suficiente (10\%). Únicamente el 8\% considera que no obtuvo aprendizaje en este nivel de estudios con respecto a la matemática.

\subsection{Percepción de la estadística, docentes y comprensión del idioma en que reciben la asignatura en la Universidad.}

En la universidad, los jóvenes perciben a los procesos de enseñanza [los docentes, en el caso de estudio, utilizan el modelo educativo basado en competencias], por parte de sus docentes, como muy claros (72\%) y el porcentaje restante consideran que son algo claros sus procesos (28\%). Respecto al aprendizaje de la estadística, perciben un buen aprendizaje (54\%), o aprendizaje regular (46\%).

En cuanto a la comprensión del idioma en que reciben las clases de estadística, español, $30 \%$ comprende siempre la clase, $28 \%$ casi siempre y $14 \%$ pocas veces. El porcentaje restante no contestó la pregunta (28\%).

\section{Discusión y conclusiones}

A nivel educativo, la enseñanza-aprendizaje exitosa en matemáticas y las disciplinas que derivan de ella, continúa siendo un desafío independientemente del nivel académico. Es necesario realizar estudios en los que pueda reflejarse la relación de esta situación con variables como grupo étnico, edad, sexo, entre otras variables. Se realizan estudios y propuestas al respecto, incluyendo la perspectiva de los estudiantes y de los docentes. En el presente estudio, el 20\% de los estudiantes entrevistados tienen lengua materna distinta al español, que es el idioma en que reciben sus clases en el sistema formal educativo.

Respecto a la comprensión del idioma en que reciben sus clases, solo 30\% afirma comprender siempre sus clases, $42 \%$ no comprenden siempre la clase (14\% la comprenden pocas veces y 28\% casi siempre). Según Jiménez et al (2010) y Jiménez y Riaño (2019) es a través de la comunicación que el estudiante construye significados, 
reflexiona, analiza y estos procesos permiten que el estudiante confronte sus ideas, partiendo que todo ello lo hace desde su lengua materna, desde la significación de su propia lengua. Es decir, los alumnos utilizan su lengua materna para construir las bases de los conceptos, incluidos los de la matemática como disciplina y en el caso de nivel universitario (Jarrett, 1999; Castillo, 2011; Lárez-Villaroel, 2018).

Por su parte, Lizarzaburu y Zapata (2001) consideran que la lengua materna es el principal instrumento de comunicación y desarrollo del pensamiento en un individuo. Es entonces que, para el caso de estudio, para la enseñanza de la matemática la lengua materna es instrumental. No obstante, en este contexto, no se cuenta con esta oportunidad de utilizar la lengua materna para la educación formal universitaria de grupo indígenas.

En este estudio, se encontró que 58\% de los entrevistados, perciben a la matemática a nivel universitario como complejas y tensas (54\%), confusas (10\%). Es importante mencionar que los porcentajes corresponden a cada característica evaluada. Respecto a los procesos de enseñanza por parte de sus docentes, $72 \%$ consideran que los procesos son muy claros, o algo claros en el caso del $28 \%$ restante.

En general, existe una enorme lista de factores determinantes para el logro exitoso del proceso de enseñanza-aprendizaje de las matemáticas a distintos niveles educativos; y se torna difícil explicar en términos sencillos las diferencias en los niveles observados en pruebas como PISA (OCDE, 2018); no obstante, como se menciona anteriormente, los factores emocionales son también un factor de importancia y en los últimos tiempos se ha tomado interés en explorar esta condición desde la investigación. Al respecto Pekrun (2014) considera que el aula es un lugar de emociones, donde los estudiantes experimentan diversos estados como entusiasmo, admiración, empatía, envidia hacia los pares, aburrimiento, persistencia, apatía, tristeza, enojo, alegría, confusión. Respecto a sus emociones, los jóvenes consideran que la matemática es atractiva (56\%), algo atractiva (38\%) y repulsiva (6\%).

Mass y Schlöglmann (2004), Mapolelo y Akinsola (2015), consideran, además, la presencia de creencias con relación a la matemática, entendiendo por creencias a las ideas respecto a la forma en que estas deben ser enseñadas ya que la población en general las considera “altamente complejas” y estas creencias tienen un impacto importante al momento de impartir la asignatura. De esta manera, las percepciones del docente respecto a la matemática y a la forma en que el estudiante recibirá y procesará la información, 
tendrán efectos en la didáctica del docente, en el ambiente y en el lenguaje como elementos fundamentales en el proceso de enseñanza-aprendizaje.

Por su parte, Luo et al (2014), así como Parker (2013), evidencian una relación entre el autoconcepto matemático, el logro y las habilidades matemáticas automatizadas.

Dentro de la constitución de los afectos, estos se producen no solo hacia las personas, sino también y de modo importante hacia los objetos y/o las situaciones en las que interactúa el ser humano, y lo importante es poder manejar estas emociones para el aprovechamiento de la vida y sus procesos (Goleman, 1996). Todo esto se manifiesta a través de las ideas, preferencias, creencias, concepciones, emociones, actitudes valores, percepciones (Ponte, 1994; Gómez, 2000; Martínez, 2005 y 2008) de ahí la importancia de haber realizado este estudio y encontrar elementos que nos hacen evidente la presencia de estos elementos desde los estudiantes universitarios hacia la matemática.

$\mathrm{Al}$ respecto y considerando la percepción y experiencias de los estudiantes universitarios, se encontró que, en los distintos niveles educativos previos a la universidad, consideran haber aprendido “algo" a nivel primaria (50\%), a nivel secundaria (54\%) y a nivel preparatoria o bachillerato (54\%).

Si bien, los resultados de este estudio muestran frecuencias relativas nada despreciables que muestran focos de alarma para la atención de la enseñanza de la matemática; es importante preguntarnos cómo se está desarrollando el proceso enseñanza-aprendizaje de esta asignatura en todos los niveles de escolaridad. Sumado a lo anterior, es necesario tomar en cuenta el análisis de las percepciones de los estudiantes, así como la presencia y naturaleza de sus emociones. Todo ello sin olvidar que existen realidades dentro del aula, que pueden ser incluso antagónicas entre sus actores sociales, docentes y estudiantes; tal es el caso de que un estudiante puede sentir repulsión o gran dificultad, por lo que está recibiendo en clase y sea únicamente por el tipo de aprendizaje que éste presenta, más auditivo que visual, o viceversa.

Es decir, se debe tomar en cuenta la diversidad cognitiva y los ritmos de aprendizaje, que bien lo explica Robles (2019) cuando considera que la educación está orientada por elementos de transformación de los paradigmas pedagógicos y didácticos: la calidad y la equidad de la educación; esto último parte del reconocimiento de la diversidad en múltiples aspectos, como lo son la diversidad cognitiva, lingüística, cultural. 
A manera de conclusión, abordar estudios que nos permitan encontrar explicaciones del por qué se dificultan los procesos de enseñanza-aprendizaje de la matemática en todos los niveles de educación formal, nos permite encontrar oportunidades de mejora. En este sentido, y apoyados en estudios previos como los realizados por Camarena Gallardo (2009), Farías y Pérez (2010), García et al (2014) podemos afirmar que los problemas que enfrentan los estudiantes no se relacionan con el agrado por la asignatura o rechazo a la misma; sino más bien, a la necesidad de estrategias pedagógicas que permitan apartarse del sentido acumulativo del conocimiento y optar por el sentido racional y lógico. Los vacíos en la comprensión de la matemática pasan de un nivel educativo a otro, y pueden llegar a ser insuperables en la educación universitaria, motivo de nuestro interés de estudio, donde la formación de futuros profesionales se ve disminuida. 


\section{Bibliografía}

Bollnow, O. (2001). Introducción a la filosofía del conocimiento. Amorrortu Editores.

Catoggio, L.M. (2007). El origen retórico del concepto de Formación en la hermenéutica gadameriana. Revista de Humanidades. Disponible en: https://www.redalyc.org/articulo.oa?id=3212/321227220004

Camarena, G.P. (2009). “La Matemática en el contexto de las ciencias”. Innovación Educativa, 9 (46), 15 - 25.

Castillo, N.M. (2011). ¿Es la comunicación un factor de aprendizaje de las matemáticas? Revista Iberoamericana de Educación. Organización de los Estados Iberoamericanos para la Educación, la Ciencia y la Cultura (OEI-CAEU).

Cerda, G., Pérez, C., Casas, J.A., y Ortega-Ruíz, R. (2017). Enseñanza y Aprendizaje de las Matemáticas: La necesidad de un análisis multidisciplinar. Psychology, Society, and Education, 9(1), 1-10 Disponible en: file://C:/Users/Ivett\%20Reyes/Downloads/DialnetEnsenanzaYAprendizajeDeLasMatematicas-6360203.pdf

Cossio, M.J.A. (2014). Pedagogía y calidad de la educación: una mirada a la formación del maestro rural. Sophia, 10(1), 14-23. Disponible en: https://www.redalyc.org/pdf/4137/413734078002.pdf

Courant, R. y Robbins, H. (1976). ¿Qué es la matemática? Ediciones Aguilar.

Devlin, M (2008). Challenging Accepted Wisdom about the Place of Conceptions of Teaching. University Teaching Improvement 18 (2), 112-119.

Dou, A. (1970). Fundamentos de la matemática. Nueva Colección Labor. Editorial Labor S.A.

Durkheim E (1979). Educación y sociología. Editorial Linotipo.

Estrada, A., Batanero, C., y Lancaster, S. (2011). Teachers’ Attitudes Towards Statistics. En Batanero, C., y Burrill, G., y Reading, C. Teaching Statistics in School Mathematics-Challenges for Teaching and Teacher Education Netherlands: Springer. 163-174. https://doi.org/10.1007/978-94-007-1131-0 
Farias, D., Pérez, J. (2010). Motivación en la enseñanza de las Matemáticas y la administración Motivación en la Enseñanza de las Matemáticas y la Administración. Formación Universitaria, 3 (6), 33-40.

Flegg, J., Mallet, D., and Lupton M. (2012). Students' perceptions of the relevance of mathematics in engineering. International Journal of Mathematical Education in Science and Technology. 43(6): 717-732.

Gadamer, H.G. (1984). Verdad y método I. Editorial Sígueme. Salamanca.

García, F.J. (2014). Presencia de la pedagogía en el acto de caminar: homo viātor, nomadismo y formación. Teoría de la Educación. Educación y Cultura en la Sociedad de la Información, 15(4): 56-84. Disponible en: https://www.redalyc.org/articulo.oa?id=2010/201032973004

García, R., García, R., y Reyes, A.J. (2014). Relación maestro alumno y sus implicaciones en el aprendizaje. $R a$ Ximhai, 10(5): 279-290. Disponible en: https://www.redalyc.org/articulo.oa?id=461/46132134019

Goleman, Daniel. (1996). La inteligencia emocional. Traducción de Elsa Mateo. Javier Vergara Editorial.

Gómez, Ch.I. (2000). Matemática emocional. Los afectos en el aprendizaje matemático. Narcea, S.A. Ediciones.

Grafe, J. (1990). Matemáticas para Economistas. Ed McGraw Hill.

Hassad, R.A. (2011). Constructivist and Behaviorist Approaches: Development and Initial Evaluation of a Teaching Practice Scale for Introductory Statistics at the College Level Education. Advancing Education in Quantitative Literacy, 4(2), $1-33$.

Jarrett, D. (1999). The inclusive classroom: Teaching mathematics and science to English language learners. It is just good teaching. 1 ed. Washington: Northwest Regional Educational Lab Portland, OR. Department of Education.

Jiménez, E.A., y Riaño, J.E. (2019). Lengua materna y comunicación en la construcción del pensamiento matemático. Bolema, Rio Claro (SP), 33(63), 248-268 
Jiménez, A., Suárez, N., y Galindo, S. (2010). La comunicación: eje en la clase de matemáticas. Praxis \& Saber, Tunja - Colombia, 1(2), 173-202.

Knijnik, G. (2007). Diversidad cultural, matemáticas y exclusión: oralidad y escrita en la educación matemática campesina del sur de Brasil. En Jiménez, J., DíezPalomar, J., y Civil, M. (Eds.). Educación Matemática y Exclusión. Guaró. España

Lárez-Villaroel, J.D. (2018). Algunos obstáculos que imposibilitan el aprendizaje efectivo de la matemática. Investigación y Postgrado 33(1), 53-74

López Moreno, L. (2010). La escuela homogeneizante. Una historia de exclusión social. Hologramática, revista de la facultad de Ciencias Sociales, UNLZ, 13 (1), 19-42 Disponible

en: http://www.cienciared.com.ar/ra/usr/3/1002/hologramatica13_v1pp19_42.pdf

Luo, W., Hogan, D., Tan, L.S., Kaur, B., Ng, P.T., y Chan, M. (2014). Self-construal and students' math self-concept, anxiety and achievement: An examination of achievement goals as mediators. Asian Journal of Social Psychology, 17, 184195.

Maass, J., y Schlöglmann, W. (2009). Beliefs and Attitudes in Mathematics Education. New Research Results Collection. Sense Publishers.

Mapolelo, D., y Akinsola, M. (2015). Preparation of Mathematics Teachers: Lessons from Review of Literature on Teachers' Knowledge, Beliefs, and Teacher Education. American Journal of Educational Research, 3(4), 505-513.

Martínez, D.B. (2002). El discurso de la educación en la diversidad en los albores del XXI. En D. Forteza \& M. R. Roselló (eds.) Educación, diversidad y calidad de vida. Palma de Mallorca: Servei de Publicacions i Intercanvi Científic. Universitat de les Illes Balears, 1-14. Disponible en https://www.nodo50.org/movicaliedu/diversidadbegona.PDF

Martínez, P.O. (2005). Dominio afectivo en Educación Matemática. Paradigma, 24(2), 7-34.

Martínez, P.O. (2008). Actitudes hacia la Matemática. Sapiens, 9(1), 237- 256. 
Martínez, P.O. (2014). Sistema de creencias acerca de la Matemática. Revista Electrónica Actualidades Investigativas en Educación, 14(3), 1-28 Disponible en https://www.redalyc.org/articulo.oa?id=447/44732048003

Nunnally, J.C. y Bernstein, I.H. (1967). Psychometric theory. Vol. 226, 3a Ed., McGrawHill.

OCDE. (2018). Programa para la Evaluación Internacional de Alumnos (PISA). PISA

Resultados. Disponible en: http://www.oecd.org/pisa/publications/PISA2018_CN_MEX_Spanish.pdf

Pamplona, R.J., Cuesta, S.J. y Cano, V.V. (2019). Estrategias de enseñanza del docente en las áreas básicas: una mirada al aprendizaje escolar. Revista Eleuthera, 21, 1333 https://doi.org/ 10.17151/eleu.2019.21.2

Parker, P.D., Marsh, H.W., Ciarrochi, J., Marshall, S., y Abduljabbar, A.S. (2013). Juxtaposing math self-efficacy and self-concept as predictors of long-term achievement outcomes. Educational Psychology, 34, 29-48.

Pekrun, R. (2014). Emotions and learning. Ginebra: The international Bureau of Education-UNESCO. Disponible en: http://www.ibe.unesco.org/fileadmin/user_upload/Publications/Educational_Pr actices/EdPractices_24eng.pdf

Ponte, J. P. (1994). Knowledge, beliefs and conceptions in mathematics teaching and learning. Proceedings of the Fifth International Conference on Systematic Co-operation between Theory and Practice in Mathematics Education , 1, 169-177

Rivera, F. y Alzate, O. (2012). La gerencia de proyectos como posibilidad de creación y transformación para el caso del especialista en gerencia educativa. Revista de Investigaciones UCM, 5(8), 115 - 126.

Robles, N.E. (2019). Práctica docente para la atención a la diversidad cognitiva: enfocado en ritmos de aprendizaje. XV Congreso Nacional de Investigación Educativa, COMIE, 2019. Área Temática 04 Procesos de Educación.

Rodríguez, R.E. (2017). La estandarización en el currículo educativo: la punta del iceberg de la homogeneización. ALTERIDAD. Revista de Educación, 12(2), 15-32. 
Sánchez BI y Camacho A (2017). Nuevos objetos y nuevas técnicas para la enseñanza de la matemática. Revista de la Escuela de Ciencias de la Educación. 12(1):115131.

Taborda, J. y Buriticá, O. (2007). El cientifismo como enfoque curricular contemporáneo. Revista Latinoamericana de Estudios Educativos, 3(1),103-122.

Uzuriaga, L., Martínez, A., y González, P. (2012). La matemática más allá de simples números y ecuaciones. Scientia Et Technica XVIII, 13(50), 112-117. Disponible en: https://www.redalyc.org/pdf/849/84923878017.pdf

Zuluaga, O., Echeverri, A., Martínez, A., Quiceno, H., Sáenz, J., y Álvarez, A. (2003). Pedagogía y epistemología. Cooperativa Editorial Magisterio.

Tuszynski, J., Sataric, M., Portet, S., y Dixon, J. (2005). Physical interpretation of microtubule self-organization in gravitational fields. Physics Letters A, 340(14), 175-180.

Villa, E., Patiño, C., Duque, L., Cardona, J.J., y Muñoz, D. (2015). Aproximación filosófico - pedagógica a la formación: Cuestiones histórico - conceptuales. Itinerario Educativo. 66(3), 281-306.

Wismath, S.L., and Worrall, A. (2015). Improving University Students' Perception of Mathematics and Mathematics Ability. Numeracy 8, Iss. 1: Article 9. DOI: http://dx.doi.org/10.5038/1936-4660.8.1.9 